\title{
Espacio de Trabajo Matemático Personal de profesores en relación a la función definida por tramos
}

\section{Personal Mathematical Working Space of teachers in relation to the Function Defined by Parts}

\section{Jesús Victoria Flores Salazar*}

(iD) https://orcid.org/0000-0002-0036-140X

$$
\text { Flor Isabel Carrillo Lara** }
$$

(iD) https://orcid.org/0000-0002-4181-3513

Tipo de Artículo: Reportes de investigación y ensayos inéditos

Doi: 10.17533/udea.unipluri.19.2.08

Cómo citar este artículo:

Salazar, J.V.F., y Carrillo, F. (2019). Espacio de Trabajo Matemático Personal de profesores en relación a la función definida por tramos. Uni-pluriversidad, 19(2),144-160. doi:10.17533/udea.unipluri.19.2.08

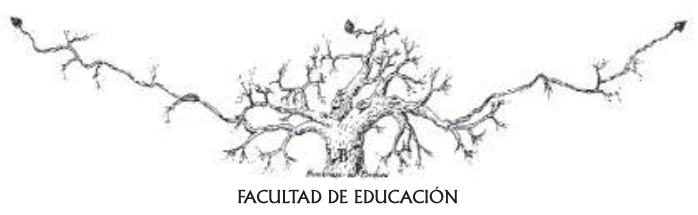

Recibido: 2019-09-23 • Aprobado: 2019-12-14

* $\quad$ Pontificia Universidad Católica del Perú. Perú

E-mail: jvflores@pucp.pe

** Pontificia Universidad Católica del Perú. Perú

E-mail: f.carrillo@pucp.edu.pe 


\title{
Resumen
}

El presente artículo es parte de una investigación que tiene como objetivo analizar el trabajo matemático personal de profesores y determinar cómo se activan las génesis semiótica, instrumental y discursiva en docentes de matemática en relación a la función por tramos. El estudio se realizó con diez profesores de matemática en un seminario-taller en Lima-Perú. Como base se utilizan aspectos teóricos y metodológicos de los Espacio de Trabajo Matemático-ETM. Como conclusiones, se evidencia en el trabajo matemático de los profesores, la activación de las génesis semiótica, instrumental y discursiva y; la activación de los planos semiótico-instrumental [Sem-Ins] e instrumental-discursivo [Ins-Dis].

Palabras clave: función por tramos, trabajo matemático, activación de planos, paradigmas.

\begin{abstract}
This paper is a part of a research that aims to analyze the personal mathematical work of teachers, and to determine how the semiotic, instrumental and discursive genesis are activated by mathematics teachers in relation to the function defined by parts. The study was conducted with ten mathematics teachers in a seminar-workshop in Lima, Perú. Theoretical and methodological aspects of the Mathematical Working Space (MWS) are used as the basis. As conclusions, it is possible to evidence personal mathematical work of the teachers, activation of semiotic, instrumental and discursive genesis, and activation of semiotic-instrumental [Sem-Ins] and semiotic-discursive [Sem-Dis] planes.
\end{abstract}

Keywords: function by parts, mathematical work, activation of planes, paradigms. 


\section{INTRODUCCIÓN}

Consideramos necesario hacer una revisión de investigaciones sobre función por tramos con el propósito de saber cuáles son las más recientes en el área de Didáctica de la Matemática, en relación con la enseñanza y el aprendizaje de la función definida por tramos. Por ello, a partir de una breve revisión de literatura, se presenta investigaciones como las de Chumpitaz (2013), Salazar y Chumpitaz (2013), Saa y Trochez (2013), Xavier (2015), Xavier y Silva (2017) y Pereira y Nascimento (2018). Estos estudios analizan desde diferentes perspectivas teóricas y diferentes niveles educativos (secundaria y superior), cómo se moviliza la noción de función definida por tramos en estudiantes y profesores.

Las investigaciones de Chumpitaz (2013) y Salazar y Chumpitaz (2013) presentan, por ejemplo, en la parte experimental una secuencia de actividades relacionadas con función por tramos y con la mediación del GeoGebra. En ambas investigaciones, los autores emplearon aspectos del Abordaje Instrumental e Ingeniería Didáctica, respectivamente. En relación con el análisis $a$ posteriori de la secuencia, en ambas investigaciones se muestra que los participantes conjeturaron, por ejemplo, que los tramos de la función no necesariamente son continuos, pues en las actividades se priorizó trabajar con representación gráfica de la función por tramos continuos y no continuos.

Por su parte, los investigadores Saa y Trochez (2013) desarrollaron un estudio con veinticuatro estudiantes de educación básica en Colombia. Para el estudio de la función por tramos con el uso del GeoGebra, diseñaron y aplicaron una secuencia de actividades con base en aspectos de los Registros de Representación Semiótica e Ingeniería Didáctica, respectivamente. Una de las conclusiones que destacamos es que el GeoGebra, como medio, proporcionó a los estudiantes la función definida por tramos mediante la comprensión de sus diferentes representaciones.

Otra investigación que se presenta en este artículo es la de Xavier (2015) y Xavier y Silva (2017). Los autores realizan una exploración que tiene como finalidad estudiar el proceso de Génesis Instrumental del artefacto función por tramos, con estudiantes de segundo y tercero año de Ensino Medio en Brasil (12 a 13 años de edad). Emplearon también aspectos del abordaje Instrumental e Ingeniería Didáctica, como bases teórica y metodológica. En dicha investigación, los autores tuvieron como finalidad comprender cómo el artefacto simbólico, función definida por tramos, se transforma en instrumento cuando se resuelve una secuencia de actividades centrada en la construcción de esta noción. En dicha secuencia, los estudiantes relacionaron la medida del área de figuras poligonales con la función por tramos. En sus conclusiones, los autores afirman que la transformación del artefacto "función por tramos" en instrumento puede ser comprobada cuando los estudiantes resuelven, de manera individual o colectiva, las actividades propuestas. Además, señalan que las acciones de los estudiantes permitieron identificar sus posibles esquemas de utilización y por consiguiente como se dio el proceso de Génesis Instrumental (Xavier, 2017, p. 123).

También, el estudio de Pereira y Nascimento (2018) tuvo como propósito investigar si construcciones realizadas en ambien- 
tes de representaciones dinámicas favorecían el aprendizaje de la función definida por tramos en intervalos reales. Los investigadores presentan una secuencia de actividades en la que utilizan el software Imagiciel. En relación con análisis de la investigación utilizaron como referencial teórico la dialéctica Herramienta-Objeto y aspectos de Ingeniería Didáctica. Los autores concluyeron que el cambio de cuadros con la mediación del Imagiciel permitió que los participantes comprendieran la noción de función por tramos, con base en la noción de dependencia e independencia de las variables de funciones reales.

Por otro lado, como la presente investigación se centra en analizar el Espacio de Trabajo Matemático-ETM personal de profesores de matemática en relación con función por tramos, se muestran también investigaciones relacionadas con la formación de profesores de matemática y especialmente, aquellas que utilizan el ETM como base teórica.

En ese sentido, la investigación de Henríquez-Rivas y Montoya-Delgadillo (2016) se centra en la vinculación de los enfoques sintético y analítico en formación de profesores de nivel secundario. En esta investigación se presenta una situación didáctica que se focaliza en el análisis del ETM personal de un futuro profesor de matemáticas y en las transiciones entre los diferentes paradigmas de la geometría de esta teoría. En esa misma línea de pensamiento, Gómez-Chacón, Botana, Escribano y Abánades (2016) proponen elementos para organizar un espacio de trabajo para problemas de lugares geométricos $\mathrm{y}$, con la interacción de software, explorar cómo futuros profesores de matemáticas perfeccionan su concepción de lugares geométricos por medio de la apropiación de las funcionalidades específicas de software de representaciones dinámicas, en relación con su propia práctica, como estudiantes y como futuros profesores de matemática.

Asimismo, la investigación de Nikolantonakis y Vivier (2016) presenta un estudio comparativo entre profesores de matemática en formación inicial de Grecia y Francia e investiga puntualmente, cómo construir un ETM idóneo para integrar las numeraciones en otras bases diferentes a la base diez. Una de las conclusiones que se relaciona con nuestro foco de interés es la que evidencia que los ETM personales e idóneos de los profesores son regidos por el sistema de numeración en base diez y que el ETM personal de profesores influye en el ETM idóneo.

Las investigaciones presentadas muestran la pertinencia del presente estudio. A continuación, presentamos los elementos teóricos y metodológicos que se utilizan en esta investigación.

\section{ELEMENTOS TEÓRICOS Y METODOLÓGICOS}

口

Utilizamos aspectos de la teoría del Espacio de Trabajo Matemático-ETM para analizar el trabajo matemático de profesores de matemática cuando resuelven una tarea sobre función definida por tramos.

Para realizar este análisis tuvimos en cuenta la noción de paradigma como conjunto de creencias, técnicas y valores compar- tidos por un grupo científico. El paradigma en el ETM determina el trabajo matemático del individuo en un dominio matemático específico como geometría, análisis, etc. Es decir, para cada dominio, el ETM define paradigmas que se pueden interpretar como la caracterización del trabajo matemático (Kuzniak, 2011). Se presentan los siguientes 
paradigmas del dominio de la geometría y del análisis:

En el dominio de la Geometría, Montoya-Delgadillo (2014), con base en Kuzniak, explicita tres paradigmas:

- Geometría natural (GI): refleja la existencia de una relación con la realidad espacial y local del individuo.

- Geometría axiomática natural (GII): la geometría es concebida como el esquema de la realidad. El razonamiento de validación se fundamenta en base a propiedades, definiciones, etc.

- Geometría axiomática formalista (GIII): los objetos geométricos provienen de una axiomática elegida con toda la rigurosidad y el formalismo del modelo geométrico elegido.
En el dominio del análisis, Montoya-Delgadillo y Vivier (2016) definen los siguientes tres paradigmas:

- Análisis Aritmético/Geométrico (AG): permite las interpretaciones y suposiciones implícitas originadas sobre la base de la geometría, de cálculos aritméticos o del mundo real.

- Análisis Calculatorio (AC): las reglas del cálculo son algo explícitas a pesar de ello, no resulta necesario reflexionar sobre la naturaleza de los objetos introducidos.

- Análisis Real (AR): involucra un trabajo matemático que implica definiciones y propiedades que son establecidas de manera teórica.

\section{Espacio de Trabajo Matemático}

El ETM tiene como aspecto fundamental el análisis del trabajo matemático que realizan tanto estudiantes como profesores. Por ello, es necesario explicar lo que desde esta teoría se llama trabajo matemático. Así, Gómez-Chacón, Kuzniak y Vivier (2016) se refieren a este como la relación del trabajo con las matemáticas. En relación al trabajo, esta idea se asocia a un grupo de actividades humanas organizadas de tal manera que permiten alcanzar los objetivos planeados, por ello, las acciones y objetivos del trabajo desde el ETM están basados en la matemática.

En relación a la tarea, Kuzniak, Tanguay y Elia (2016) explican que una tarea es considerada como cualquier tipo de problema matemático, con preguntas establecidas de manera explícita y clara, que requiere de un tiempo predecible para su resolución. Así mismo, la teoría ETM ha sido desarrollada buscando vincular y preservar los planos epistemológico y cognitivo. El plano epistemológico está centrado en el contenido matemático con una reflexión sobre su organización mientras que, el plano cognitivo se focaliza en los aspectos visibles y tangibles de la actividad desarrollada por el sujeto.

Estos dos planos están organizados de manera triádica. En el plano epistemológico se encuentra el representamen (objetos concretos y tangibles); el artefacto (regla, compás, fórmulas matemáticas, etc.); y el referencial teórico (definiciones, propiedades y teoremas). El plano cognitivo es constituido por la visualización (interpretación de los signos); la construcción (artefactos y técnicas) y las pruebas (validaciones con base en el referencial teórico). La articulación de los planos epistemológico y cognitivo es dada por tres génesis, como se muestran en el diagrama del ETM en la Figura 1. 


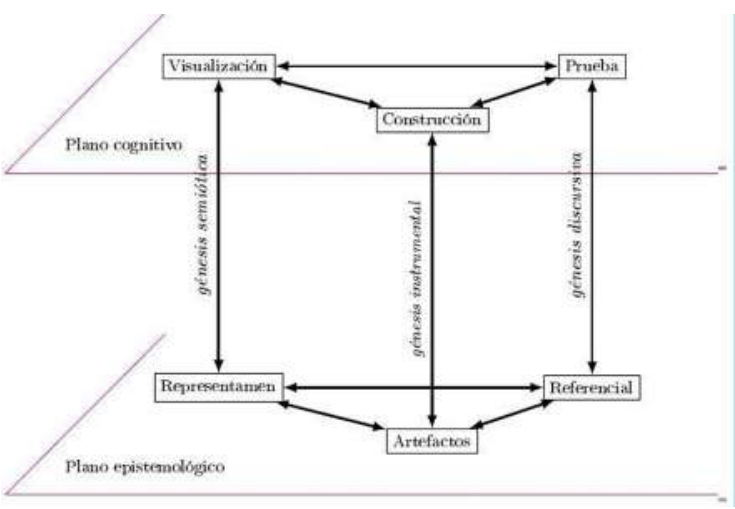

Figura 1: Diagrama del ETM (Gómez-Chacón et al., 2016, p.6)

Así, en la Figura 1 se presenta una génesis semiótica que articula representaciones semióticas con la visualización; una génesis instrumental que permite hacer operatorios los artefactos para la construcción y que contribuyen a alcanzar el trabajo matemático y; una génesis discursiva que vincula el referencial teórico con la prueba utilizando las propiedades del sistema del referencial teórico para ponerlas al servicio del razonamiento matemático y para su validación.

Por otro lado, todas las interacciones entre los planos epistemológico y cognitivo y las génesis, se fusionan en tres planos verticales: Semiótico-Instrumental [Sem-Ins], Instrumental-Discursivo [Ins-Dis] y Semiótico-Discursivo [Sem-Dis], como se muestra en la Figura 2.

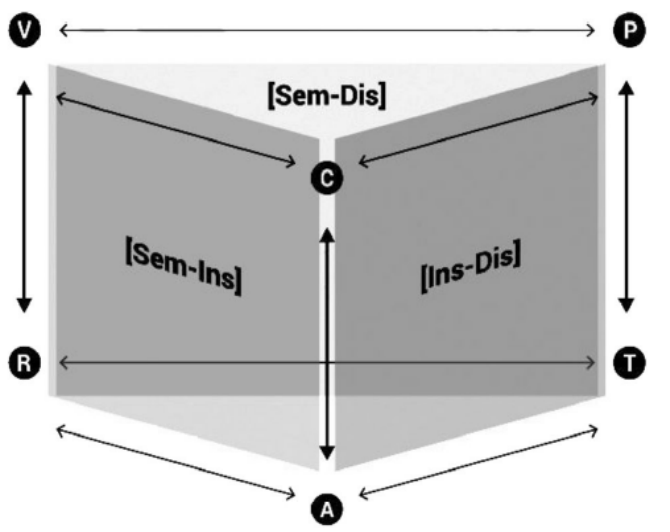

Figura 2: Planos verticales del ETM (Kuzniak, Tanguay y Elia (2016, p.726)
En el plano Semiótico-Instrumental [Sem-Ins] se distinguen dos formas de trabajo matemático, una orientada hacia la construcción de los resultados (figuras, gráficos) que cumplen algunas condiciones, y otra orientada hacia la interpretación de los datos brindados por el/los artefactos. En el plano Instrumental-Discursivo [Ins-Dis] el punto crucial es la prueba, que puede ser una justificación, una prueba experimental o la argumentación deductiva pura. El plano Semiótico-Discursivo [Sem-Dis] se basa en propiedades, signos y la visualización; desempeña un papel heurístico pues hace visible ciertos estados y contextos del trabajo matemático.

En relación con la activación de las génesis y/o los planos verticales, en el sentido del ETM, una o varias génesis se activan un sujeto relaciona las triadas del plano epistemológico (representamen, artefacto y referencial) al resolver una tarea y las asocia a las del plano cognitivo (visualización, construcción y prueba). En ese mismo sentido, uno o varios planos verticales se activan cuando dos o más génesis se activan.

Otra particularidad que es necesario presentar en relación con la teoría del ETM, de acuerdo con Kuzniak, Tanguay y Elia (2016), son los tipos de ETM llamados: ETM de referencia, ETM idóneo y ETM personal.

[...] de esta manera, el trabajo matemático en un marco escolar se puede describir gracias a estos tres niveles de ETM: la matemática considerada por la institución que se describe en el ETM de referencia, éste es desarrollado por el profesor hasta alcanzar un ETM idóneo que permita un establecimiento efectivo en clase, donde cada alumno trabaja en su ETM personal (Gómez-Chacón et al., 2016, p. 12). 
Así mismo, los autores establecen que para el trabajo matemático en el contexto escolar, también se tiene que tomar en cuenta las orientaciones curriculares de las instituciones educativas.

\section{Aspectos Metodológicos y LA TAREA}

Se señala que la presente investigación muestra una visión ampliada del trabajo de Salazar y Carrillo (2019).

La investigación se realizó en el marco de un seminario-taller realizado en mayo del 2018 en una universidad particular de Lima, Perú. El seminario-taller constó de dos sesiones de 90 minutos cada una, durante dos sábados consecutivos. Además, se contó con un aula y un laboratorio de informática en el que está instalado el software GeoGebra.

En el seminario-taller participaron diez docentes de matemática de nivel secundaria (Educación Básica Regular) que tienen entre 4 y 7 años de experiencia docente y enseñan en instituciones educativas nacionales y particulares de Lima. Sin embargo, de los diez profesores participantes, para este estudio fueron seleccionados, de manera aleato- ria, dos profesores a los que denominaremos Profesor A y Profesor B para resguardar la identidad de cada uno (de acuerdo al protocolo de consentimiento informado elaborado para esta investigación, según los lineamientos del comité de ética). En cuanto a la recolección de los datos, se recolectaron las producciones de los profesores en sus fichas de trabajo y archivos GeoGebra.

A continuación, se presenta la tarea en la Figura 3. Cabe resaltar que esta tarea permite realizar un trabajo matemático en dos dominios, en el dominio de la geometría (figuras planas y área) y en el dominio del Análisis (función por tramos), y tiene como finalidad analizar la activación de las génesis semiótica, instrumental y discursiva, en relación con la función por tramos, en el en el Espacio de Trabajo Matemático de profesores de matemática.

Como se muestra en la figura, un punto $M$ recorre el lado de un rectángulo $A B C D$ de 4 u.m. de largo y 2 u.m. de ancho. Llamemos " $x$ " a la longitud del recorrido de $A$ a $M$.
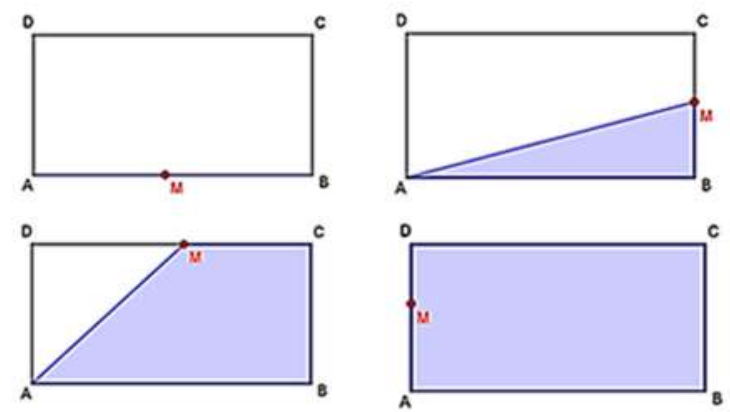

En base a esta información: exprese la medida del área de la parte sombreada de celeste, con respecto a la distancia recorrida del punto $M$ y represéntelo gráficamente.

Figura 3: Tarea sobre función definida por tramos (Adaptada de Xavier, 2015) 
En la tarea se espera que los profesores movilicen la noción de la función por tramos, con base en la medida del área de distintos polígonos que se originan cuando el punto $\mathrm{M}$ recorre los diferentes lados del rectángulo dado, y además, que interpreten la regla de correspondencia de la función por tramos definida a partir de una variable real. Es por ello que se espera que el trabajo matemático personal de los profesores esté caracterizado en los paradigmas GII del dominio de la geometría y de los paradigmas del análisis Aritmético/Geométrico (AG) y del Análisis Calculatorio (AC).

En seguida, se presenta lo que se espera que los participantes del seminario-taller realicen "a priori", con base en los aspectos teóricos y metodológicos del ETM.

En un primer trayecto, cuando el punto pertenece al lado del rectángulo, la medida del área es cero, pues no existe parte sombreada. En relación al recorrido del punto $\mathrm{M}$ por el lado $\mathrm{BC}$ del rectángulo $\mathrm{ABCD}$ (segundo trayecto), se observa que conforme el punto recorre el lado $\mathrm{BC}$, la medida del área sombreada es la medida del área de un triángulo rectángulo ABM.

En un tercer trayecto, cuando el punto $\mathrm{M}$ recorre el lado $\mathrm{CD}$ del rectángulo $\mathrm{ABCD}$, la medida del área será dada por la medida del área del trapecio rectangular ABCM. Finalmente, cuando el punto $\mathrm{M}$ recorre el lado AD del rectángulo (cuarto trayecto), la medida del área de la figura sombreada será dada por la medida del área del rectángulo ABCD.

Notemos que se ha relacionado la medida del área de la parte sombreada con cuatro trayectos y que se está asociando todo el trabajo matemático en dos dominios (geometría y análisis), pues la tarea solicita; además, que se represente gráficamente la medida del área de acuerdo con el recorrido del punto M. En ese sentido, si llamamos " $x$ " a la longitud del recorrido de $\mathrm{M}$, los cuatro trayectos se sintetizarían de la siguiente manera:

$$
\begin{aligned}
& f(x)=0 ; \quad 0 \leq x \leq 4 \\
& f(x)=\frac{(4)(x-4)}{2}=2(x-4)=(2 x-8), \text { cuando } 4<x \leq 6 \\
& f(x)=\frac{(4+x-6)(2)}{2}=\frac{(x-2)(2)}{2}=(x-2), \text { cuando } 6<x<10 \\
& f(x)=(4)(2)=8, \text { cuando } 10 \leq x \leq 12
\end{aligned}
$$

Teniendo en cuenta la presentación anterior, se determina que la medida del área de la parte sombreada en relación al recorri- do del punto $\mathrm{M}$ por los lados del rectángulo $\mathrm{ABCD}$, define a una función por tramos cuya regla de correspondencia es la siguiente:

$$
f(x)=\left\{\begin{array}{lr}
0, & \text { si } 0 \leq x \leq 4 \\
2 x-8, & \text { si } 4<x \leq 6 \\
x-2, & \text { si } 6<x<10 \\
8, & \text { si } 10 \leq x \leq 12
\end{array}\right.
$$


La Figura 4 muestra la representación figural y gráfica de los cuatro trayectos explicados anteriormente.

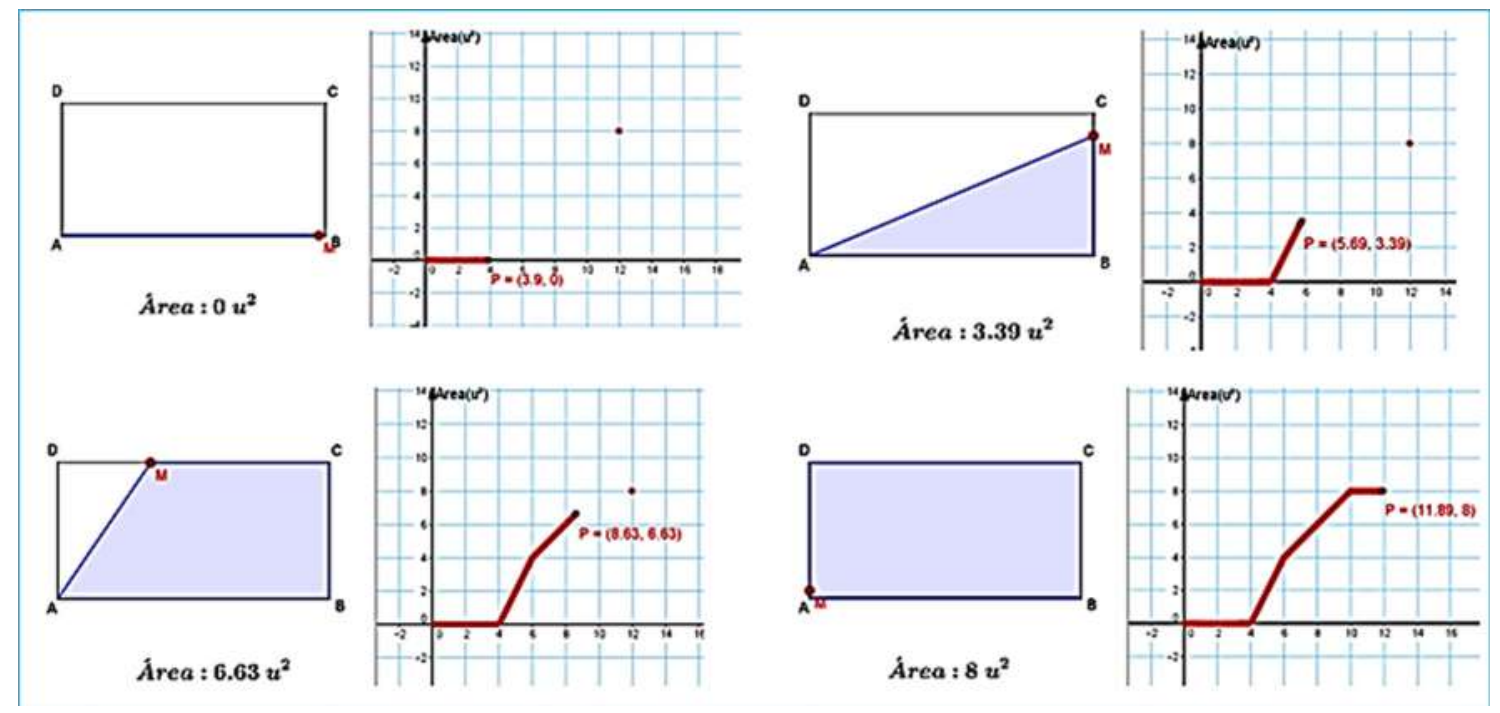

Figura 4: Representación figural y gráfica de la tarea

Como se muestra en la Figura 4, las dos ventanas del GeoGebra promueven la coordinación de las representaciones figural y gráfica y movilizan la noción de función definida por tramos en diferentes dominios matemáticos.

De acuerdo con la tarea presentada, se muestra la producción de los dos profesores seleccionados (Profesor A y Profesor B) y sus respectivos trabajos realizados en GeoGebra.

\section{Trabajo matemático personal del profesor $A$}

El profesor A identifica el recorrido del punto $\mathrm{M}$ en cuatro pasos que se muestra a continuación (ver Tabla 1) y utiliza el GeoGebra (artefacto) para representar lo solicitado en la ficha de trabajo.

Tabla 1: Desarrollo de la tarea-profesor A

\begin{tabular}{|c|c|c|}
\hline paso 1 & $\begin{array}{l}a(x)=0(u \cdot m)^{2} \\
\text { En este primer gráfico, el } \\
\text { desplazamiento del punto M } \\
\text { describe el segmento AM de langitud } \\
x \mathrm{~cm} \text {; por definición de área se } \\
\text { establece que el área en este punto } \\
\text { es igual a cero. }\end{array}$ & 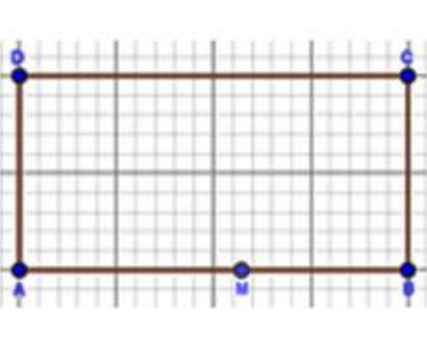 \\
\hline paso 2 & 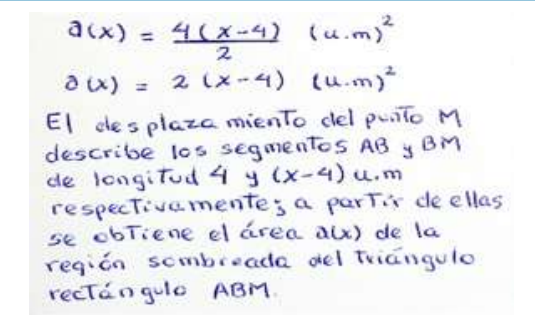 & 4 \\
\hline
\end{tabular}




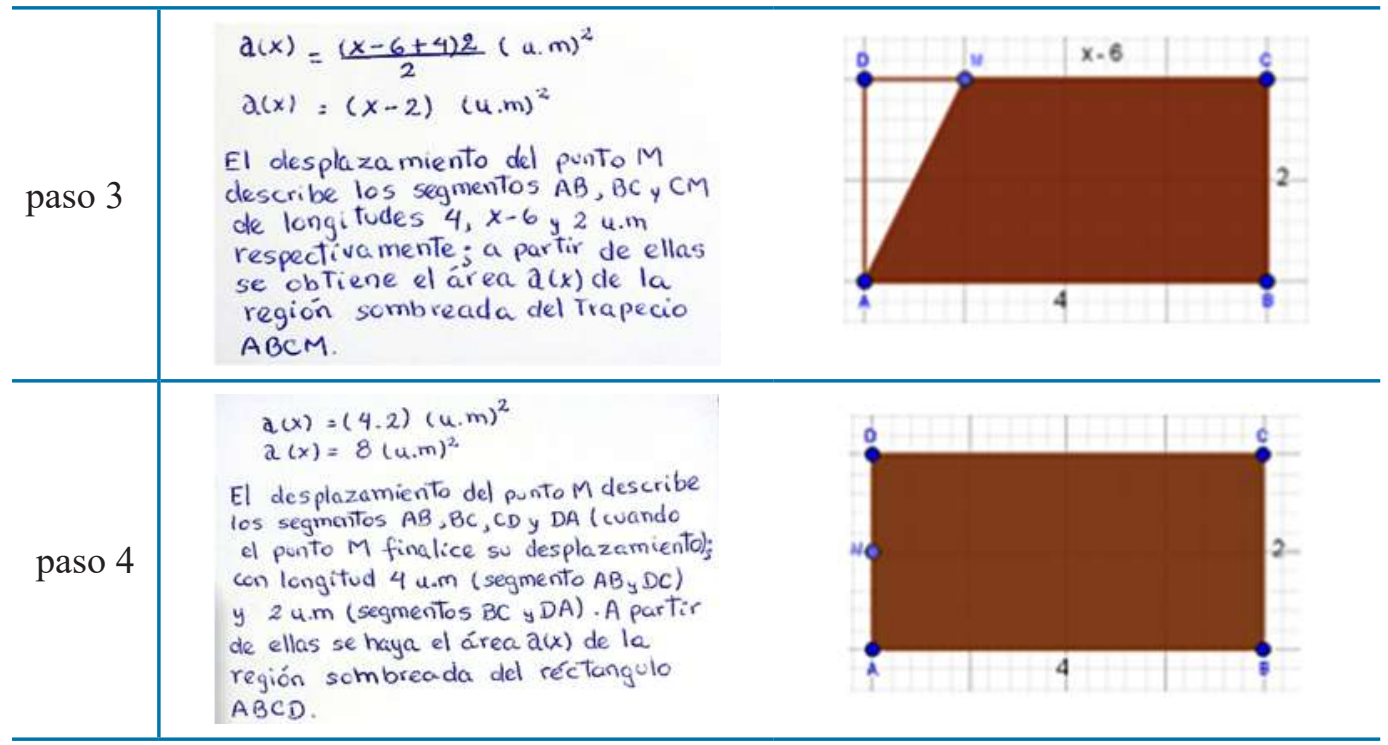

En este procedimiento se puede observar que el profesor A inicia su trabajo matemático en el dominio de la Geometría, pues en los cuatro pasos hace referencia a elementos geométricos como segmentos, área de la región sombreada, polígonos (triángulo rectángulo, trapecio y rectángulo).

Luego, hace un cambio de dominio y efectúa conversiones y tratamientos algebraicos (tratamientos algebraicos para determinar la expresión área según una variable $\mathrm{x}$, conversiones de la representación figural a la representación algebraica). De acuerdo con el ETM, esto evidencia que la génesis semiótica se activó ya que representa una función por tramos (ver figura 5), lo que hace suponer que asoció los aspectos geométricos con los del dominio del análisis.

$$
\begin{aligned}
& \text { Finalmente, setiene: } \\
& a(x)=\left\{\begin{array}{cc}
0, & 0 \leqslant x \leqslant 4 \\
2 x-8, & 4<x \leqslant 6 \\
1 x-1, & 6<x \leqslant 10 \\
8, & 10<x \leqslant 12
\end{array}\right.
\end{aligned}
$$

Figura 5: Conclusión de la tarea-profesor A

En cuanto al trabajo matemático, este es semejante al esperado "a priori" pues determina la regla de correspondencia de la función por tramos y después de ello, para representarla gráficamente utiliza el GeoGebra, como se muestra en la Figura 6. Sin embargo, sus acciones muestran que el profesor A está instrumentalizado en relación al artefacto GeoGebra (activación de la génesis instrumental), ya que no solo representa la gráfica de la función por tramos, sino que la asocia con la representación figural, lo que muestra que articula los dominios de la geometría y del análisis.

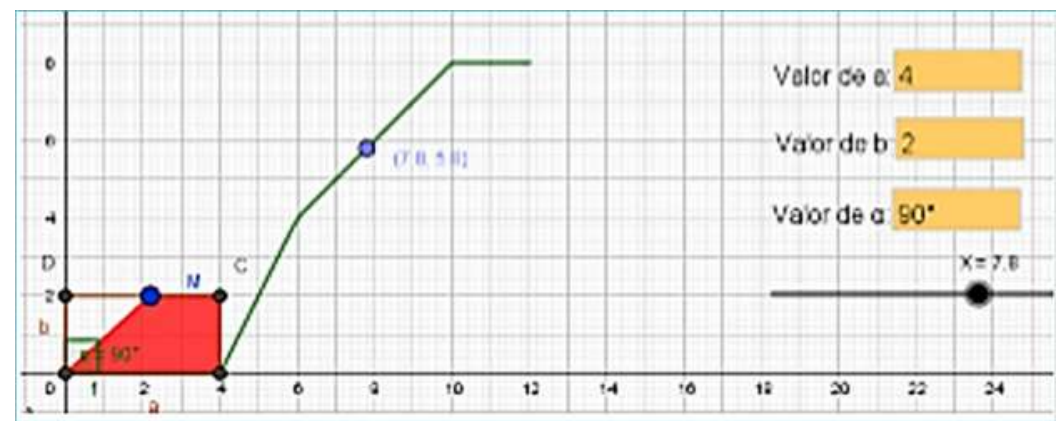

Figura 6: Representación gráfica-profesor A 
A partir de la Figura 6, se manifiesta la activación de la génesis instrumental, ya que se emplea el GeoGebra como artefacto en el proceso constructivo en el trabajo y muestra la activación del plano [Sem-Ins].

Con respecto a los paradigmas que se identifican en el trabajo realizado por el profesor A, mencionamos los siguientes. En los cuatro pasos el profesor manifiesta características del trabajo matemático en el paradigma de la geometría GII, debido a que menciona objetos geométricos (segmento, triángulo rectángulo, trapecio y rectángulo); además, el profesor emplea la definición de área y valida dichos conceptos basado en definiciones propias del dominio. Con respecto al dominio Análisis, en primer lugar, su trabajo está caracterizado en el paradigma Análisis Aritmético-Geométrico (AG) ya que, a partir de conceptos de la geometría (como longitud de segmentos y área de polígonos), realiza reglas de correspondencia según cada tramo recorrido por el punto $\mathrm{M}$, de acuerdo con la tarea. En segundo lugar, es posible identificar características del paradigma Análisis-Calculatorio (AC), ya que se basa en reglas del cálculo ya definidas y expresa la función tramos en una variable real.

En la Tabla 2 se sintetiza el trabajo matemático del profesor A en relación con las diferentes génesis y la activación de los planos del ETM.

Tabla 2: Síntesis del trabajo matemático del profesor A

\begin{tabular}{|c|c|c|}
\hline Trabajo matemático & Tipo de génesis & $\begin{array}{c}\text { Planos } \\
\text { activados }\end{array}$ \\
\hline $\begin{array}{l}\text { Identifica el recorrido del punto } \mathrm{M} \\
\text { en la representación figural, además } \\
\text { denota } \mathbf{a}(\mathbf{x})=\mathbf{0} \text {. }\end{array}$ & $\begin{array}{l}\text { Génesis semiótica y discursiva: } \\
\text { Emplea la representación figural para in- } \\
\text { terpretar el recorrido del punto. } \\
\text { También emplea la representación alge- }\end{array}$ & \multirow{3}{*}{ [Sem-Ins] } \\
\hline $\begin{array}{l}\text { Calcula la medida de área de los polí- } \\
\text { gonos (triángulo y trapecio) formados } \\
\text { según cada trayecto recorrido por el } \\
\text { punto M. }\end{array}$ & $\begin{array}{l}\text { braica. } \\
\text { Génesis instrumental: } \\
\text { Identifica y emplea las definiciones de } \\
\text { áreas de polígonos (artefacto simbólico). }\end{array}$ & \\
\hline $\begin{array}{l}\text { Utiliza la fórmula de la medida de } \\
\text { áreas de polígonos. }\end{array}$ & $\begin{array}{l}\text { Génesis instrumental: } \\
\text { Uso de herramientas algebraicas como } \\
\text { artefacto simbólico. }\end{array}$ & \\
\hline $\begin{array}{l}\text { Determina la medida del área de los } \\
\text { polígonos como una función, según } \\
\text { cada tramo. }\end{array}$ & $\begin{array}{l}\text { Génesis semiótica: } \\
\text { Realiza conversiones y tratamientos alge- } \\
\text { braicos (figural y algebraica) que le per- } \\
\text { mite obtener el resultado deseado. } \\
\text { Génesis instrumental: } \\
\text { Moviliza la definición de función definida } \\
\text { por tramos (permite que el artefacto sea } \\
\text { operatorio). }\end{array}$ & [Ins-Dis] \\
\hline $\begin{array}{l}\text { Representa gráficamente la función } \\
\text { por tramos empleando el ambiente } \\
\text { de representaciones dinámicas Geo- } \\
\text { Gebra. }\end{array}$ & $\begin{array}{l}\text { Génesis semiótica: } \\
\text { Coordina las representaciones algebraica } \\
\text { y gráfica para determinar la función por } \\
\text { tramos. }\end{array}$ & [Sem-Ins] \\
\hline
\end{tabular}


Seguidamente, se presenta el trabajo matemático del profesor B.

Trabajo matemático personal del profesor B
Para la descripción y análisis del desarrollo de la tarea del profesor B consideramos su ficha y archivo en GeoGebra. La Tabla 3, presenta el paso a paso del trabajo matemático del profesor.

Tabla 3: Desarrollo de la tarea-profesor B

\begin{tabular}{|c|c|}
\hline paso 1 & $\begin{array}{l}\text { i) En la primera figura, el punto } M \text { sdo se desplasa } \\
\text { sobre el segmento } A B \text {, dicha trayectoria origha el } \\
\text { segmento } A M \text { de área nula. } \\
\text { Entonces } a(x)=0 \text {, si } 0 \leq x \leq 4\end{array}$ \\
\hline paso 2 & $\begin{array}{l}\text { ii) En la segunda figura, el junto } M \text { solo se desplaza } \\
\text { sobre el segmento } B C \text {, dicha trayectoria origina } \\
\text { el triángulo } A B M \text { cyá área os: } \\
\left\{\begin{array}{l}A B=4 \\
B M=x-4\end{array} \Rightarrow \text { área }=\frac{4 \times(x-4)}{2}=2 x-8\right. \\
\text { Entones } a(x)=2 x-8 \text { si } 4<x \leqslant 6\end{array}$ \\
\hline paso 3 & $\begin{array}{l}\text { iii) En la tercera figura, el punto } M \text { solo se desplaza } \\
\text { sobre el segmento } C D \text {, dicha trayectoria origina el } \\
\text { trapecio } A B C M \text {, cuya área es: } \\
\left\{\begin{array}{l}A B=4 \\
B C=2 \\
C M=x-6\end{array} \Rightarrow \text { área }=\left(\frac{4+(x-6)}{2}\right) \times 2=x-2\right. \\
\text { Entonces } a(x)=x-2 \quad \text { Si } 6<x \leqslant 10\end{array}$ \\
\hline paso 4 & $\begin{array}{l}\text { iv) En la cuarta figura, el punto } M \text { solo se desplaza } \\
\text { sobre el segmento } D A \text {, dicha trayectoria origina el } \\
\text { rectángulo } A B C D \text { cuya áred of } 8 \mathrm{um}^{2} \\
\text { Entonces } a(x)=8 \quad \text { si } 10<x \leqslant 12\end{array}$ \\
\hline paso 5 & 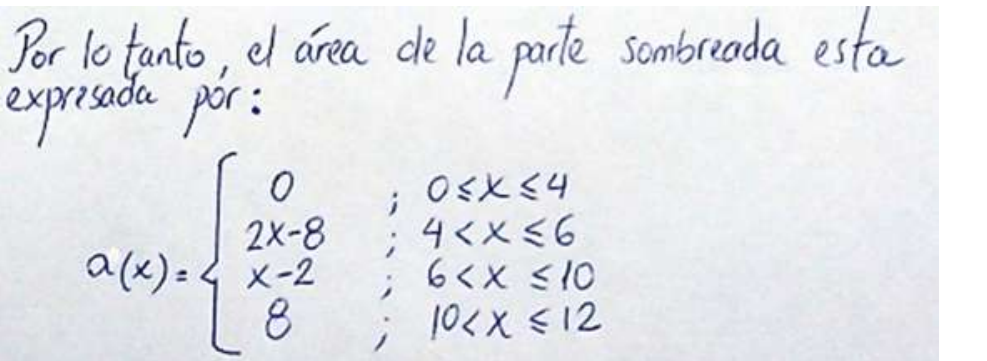 \\
\hline
\end{tabular}

El profesor B expresa en lenguaje natural el comportamiento del recorrido del punto $\mathrm{M}$ y lo representa algebraicamente para cada tramo, es decir, al igual que el profesor A, se encuentra en el dominio de la geometría ya que menciona términos como segmento, área, etc. Luego, efectúa conversiones y tratamientos algebraicos de manera explícita ya que relaciona la medida de cada segmento y define el área de cada polígono 
identificado según los tramos de la función (ver tabla 3). Por lo tanto, se determina la activación de la génesis semiótica.

Además, identificamos que cada paso concluye con una regla de correspondencia en función de una variable $\mathrm{x}$, es decir, se ha realizado un cambio de dominio. Este cambio es del dominio de la geometría al dominio del análisis, ya que finalmente representa algebraicamente una función por tramos de una variable real.

En cuanto al trabajo matemático, al realizar la representación gráfica de la función por tramos, el profesor B emplea el GeoGebra. En el sentido del ETM, se activa la génesis instrumental (ver figura 7).

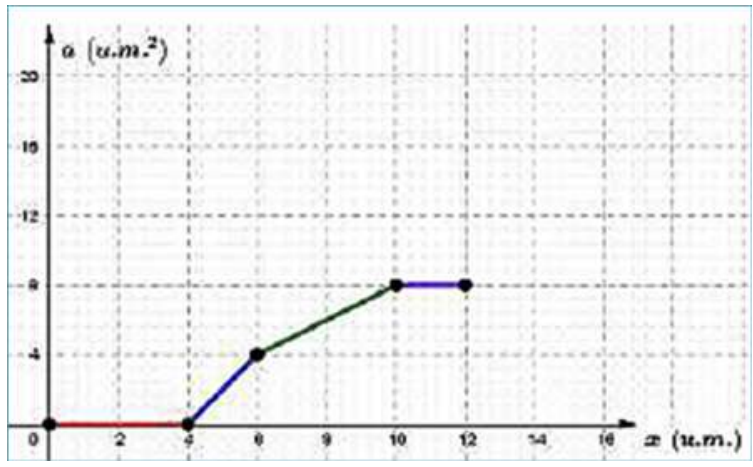

Figura 7: Representación gráfica-profesor $B$
En cuanto a la representación de la Figura 7, el profesor B emplea un color diferente para cada tramo. Además, las acciones del profesor al desarrollar la tarea evidencian que coordinó las representaciones algebraica y gráfica.

Con respecto a los paradigmas del ETM que caracterizan el trabajo realizado por el profesor B, mencionamos los siguientes. En el dominio Análisis, su trabajo presenta características del paradigma Análisis Aritmético-Geométrico (AG) porque emplea conceptos de medida de un segmento, área de un triángulo, área de un trapecio y área de un rectángulo. A partir de estos conceptos de la geometría presenta distintas reglas de correspondencia según cada tramo recorrido por el punto $\mathrm{M}$, en el que define una variable $\mathrm{x}$. Posteriormente, podemos identificar características del paradigma Análisis-Calculatorio (AC), ya que el profesor B se basa en reglas del cálculo ya definidas y expresa la función tramos en una variable real.

En la Tabla 4 se presenta el trabajo matemático del profesor $\mathrm{B}$ en relación con las diferentes génesis y la activación de los planos del ETM.

Tabla 4. Análisis del trabajo matemático del profesor B.

\begin{tabular}{|c|c|c|}
\hline Trabajo matemático & Tipo de génesis & $\begin{array}{c}\text { Planos } \\
\text { activados }\end{array}$ \\
\hline $\begin{array}{l}\text { Identifica el recorrido del punto y a } \\
\text { dicha longitud la denomina con la va- } \\
\text { riable x. }\end{array}$ & $\begin{array}{l}\text { Génesis semiótica: } \\
\text { Usa representaciones para definir la } \\
\text { variable } \mathrm{x} .\end{array}$ & \\
\hline $\begin{array}{l}\text { Relaciona el recorrido del punto con la } \\
\text { medida de las áreas formadas por cada } \\
\text { tramo. }\end{array}$ & $\begin{array}{l}\text { Génesis instrumental y discursiva: } \\
\text { Emplea las definiciones de áreas de } \\
\text { polígonos (artefacto simbólico). }\end{array}$ & [Sem-Ins] \\
\hline $\begin{array}{l}\text { Utiliza las fórmulas de la medida de las } \\
\text { áreas de los polígonos. }\end{array}$ & $\begin{array}{l}\text { Génesis instrumental: } \\
\text { Utiliza las fórmulas como artefacto } \\
\text { simbólico. }\end{array}$ & \\
\hline
\end{tabular}


Trabajo matemático

\begin{tabular}{lll} 
& \multicolumn{1}{c}{ Tóno de génesis } & activados \\
\hline $\begin{array}{lll}\text { Determina la regla de correspondencia } \\
\text { de la función definida por tramos. }\end{array}$ & $\begin{array}{l}\text { Génesis semiótica: } \\
\text { En la representación algebraica, calcu- }\end{array}$ & \\
& $\begin{array}{l}\text { la la longitud "x" (recorrido según el } \\
\text { punto . }\end{array}$ & [Sem-Ins] \\
\hline $\begin{array}{l}\text { Representa gráficamente la función por } \\
\text { tramos con el uso del GeoGebra. }\end{array}$ & Génesis instrumental: & \\
& Moviliza la definición de función por & [Ins-Dis] \\
& tramos, coordina el registro algebraico & \\
& y gráfico (instrumento semiótico). & \\
\hline
\end{tabular}

Como se evidencia en la Tabla 4, en relación con el dominio de la geometría, el profesor manifiesta características del paradigma Geometría Axiomática Natural (GII), debido a que menciona objetos geométricos (segmento, triángulo rectángulo, trapecio y rectángulo). Además, el profesor emplea la definición de área y valida los conceptos basado en definiciones propias del dominio.

\section{DISCUSIÓN Y REFLEXIÓN FINAL}

Al resolver la tarea sobre función definida por tramos que guarda relación con la medida del área de un rectángulo dado, los profesores activan sus conocimientos previos (por ejemplo, sobre medida de áreas y función) y sus acciones muestran el ETM personal de cada uno. Esto evidencia que convergen los elementos epistemológicos y cognitivos en la resolución de dicha tarea.

También, en el sentido del Espacio de Trabajo Matemático, hemos identificado los paradigmas que caracterizan el trabajo realizado por los profesores al resolver la tarea. Estos son fundamentalmente los paradigmas GI y GII del dominio de la Geometría y los paradigmas AG y AC del dominio del Análisis.

En relación con el trabajo matemático de los profesores al resolver la tarea, se evidencia la activación de las génesis semiótica, instrumental y discursiva, además, sus acciones permiten identificar la activación de los dos planos verticales Semiótico-Instrumental e Instrumental-Discursivo del
ETM. Si bien los participantes utilizan sus herramientas teóricas para desarrollar la tarea, se observa la preponderancia semiótica e instrumental. En relación con el artefacto simbólico fórmula de medida de área, ambos profesores lo utilizan para determinar cada tramo de la función y se evidencia tratamientos algebraicos.

Por otro lado, en cuanto a la representación gráfica de la función definida por tramos en la que los profesores utilizan el GeoGebra, se observa que el profesor A está instrumentalizado con el artefacto GeoGebra, ya que consigue representar la función definida por tramos, asociada a la medida del área del rectángulo. Por su parte, a pesar de utilizar el GeoGebra, el profesor B no está necesariamente instrumentalizado con este artefacto, pues la gráfica muestra que no está apropiado de éste.

En relación con las investigaciones de la revisión de literatura, Chumpitaz (2013) y Salazar y Chumpitaz (2013) afirman que los participantes conjeturaron sobre la con- 
tinuidad de la función por tramos. Sin embargo, esto no ocurrió en nuestra investigación porque en ningún caso los profesores relacionaron el trabajo matemático con las características de continuidad que presenta dicha función.

En cuanto al uso del GeoGebra, Saa y Trochez (2013) concluyen que el software es un medio que proporciona la comprensión de la función definida por tramos en sus diferentes representaciones. En nuestra investigación verificamos que los profesores emplearon diferentes representaciones: la representación algebraica y figural.

Con respecto a la tarea, se tomó como base los trabajos de Xavier (2015) y Xavier y Silva (2017) para realizar una adaptación cuyo análisis se hizo desde el Espacio de Trabajo Matemático.
Al igual que el estudio de Pereira y Nascimento (2018), podemos concluir que el cambio de dominios matemáticos (geometría y análisis) propiciado por el software GeoGebra permitió que los participantes comprendieran la noción de función por tramos con base en la noción de dependencia e independencia de las variables de funciones reales.

De manera similar que en la investigación de Gómez-Chacón, Botana, Escribano y Abánades (2016), en este estudio proponemos elementos para organizar un trabajo matemático en los dominios del Análisis y de la Geometría, relacionados con la interacción del GeoGebra cuando profesores de matemáticas en servicio mejoran su concepción de función definida por tramos, por medio de la apropiación de las funciones específicas del software.

\section{Agradecimientos}

A la Pontificia Universidad Católica del Perú, especialmente a la Dirección de Gestión de la Investigación-DGI en el marco del proyecto ID-575. Al mismo tiempo, a la lí- nea de investigación Tecnologías y Visualización en Educación Matemática-TecVEM por el apoyo brindado en la presente investigación.

\section{Nota}

1. El presente artículo es resultado del proyecto "Articulación de dominios matemáticos por medio de la modelización y la tecnología digital en profesores de matemática" ID 575-2018, financiado por la Dirección de Gestión de la Investigación-DGI de la Pontificia Universidad Católica del Perú.

\section{REFERENCIAS BIBLIOGRÁFICAS}

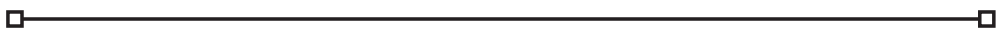

Chumpitaz, L. (2013). La Génesis Instrumental: Un estudio de los procesos de instrumentalización en el aprendizaje de la función definida por tramos mediado por el software GeoGebra con estudiantes de ingeniería. Tesis (maestría en Enseñanza de las Matemáticas), Pontificia Universidad Católica del Perú, Lima, Perú. Recuperado de http://tesis.pucp.edu.pe/repositorio/handle/20.500.12404/4514 
Gómez-Chacón, I. M., Botana, F., Escribano, J., y Abánades, M. Á. (2016). Concepto de Lugar Geométrico. Génesis de Utilización Personal y Profesional con Distintas Herramientas. Bolema: Boletim de Educação Matemática, 30(54), 67-94. doi: 10.1590/1980-4415v30n54a04

Gómez-Chacón, I., Kuzniak, A., y Vivier, L. (2016). El rol del profesor desde la perspectiva de los Espacios de Trabajo Matemático. Boletim de Educação Matemática, 30(54), 1-22. doi: 10.1590/1980-4415v30n54a01

Henríquez-Rivas, C., y Montoya-Delgadillo, E. (2016). El Trabajo Matemático de Profesores en el Tránsito de la Geometría Sintética a la Analítica en el Liceo. Boletim de Educação Matemática, 30(54), 45-66. doi :10.1590/1980-4415v30n54a03

Kuzniak, A. (2011). L'espace de Travail Mathématique et ses genèses. Annales de didactique et de sciences cognitives, 16, 9-24.

Kuzniak, A., Tanguay, D., y Elia, I. (2016). Mathematical Working Spaces in schooling: An introduction. ZDM-The International Journal on Mathematics Education, 48(6), 721-737. doi: 10.1007/ s11858-016-0812-x

Nikolantonakis, K., y Vivier, L. (2016). El ETM de Futuros Profesores de Primaria en un Trabajo sobre los Números Naturales en Cualquier Base. Boletim de Educação Matemática, 30(54), 23 44. doi:10.1590/1980-4415v30n54a02

Montoya-Delgadillo, E., y Vivier, L. (2014). El proceso de prueba en el espacio de trabajo geométrico: profesores en formación inicial. Enseñanza de las Ciencias, 32(3), 225-244. doi:10.5565/ rev/ensciencias. 1049

Montoya-Delgadillo, E., y Vivier, L. (2016). Mathematical working space and paradigms as an analysis tool for the teaching and learning of analysis. ZDM-The International Journal on Mathematics Education, 48(6), 739-754. doi: 10.1007/s11858-016-0777-9

Pereira, C., y Nascimento, H. (2018). Proposta de estudo de função mediada pelo GeoGebra. Acta Scientiae, 20(1), 75 - 94. doi: 10.17648/acta.scientiae.v20iss1id3831

Saa, A., y Trochez, Á. (2013). Una propuesta de enseñanza de la función por tramos usando el periódico y GeoGebra. Tesis (Licenciatura en Educación) Universidad del Valle, Cali, Colombia. Recuperado de http://tesis.pucp.edu.pe/repositorio/handle/123456789/4514

Salazar, J.V.F., y Chumpitaz, D. (2013). Génesis instrumental: un estudio del proceso de instrumentalización de la función definida por tramos. Actas del VII Congreso Iberoamericano de Educación Matemática - VII CIBEM. Sociedad de Educación Matemática del Uruguay, pp. 6863 - 6870.

Salazar, J.V.F., y Carrillo, F.I. (2019). Función definida por tramos: espacio de trabajo matemático y su relación con las representaciones semióticas. Actas del VI Simposio sobre el Trabajo Matemático-ETM6. Pontificia Universidad Católica de Valparaíso, pp.219-231.

Xavier Neto, A. L. (2015). Um estudo da Gênese Instrumental para função de uma variável real com várias sentenças. Tesis (maestria en Educación Matemática). Pontifícia Universidad Católica de São Paulo, São Paulo, Brasil. 
Xavier Neto, A. L., y Silva, M. J.F. (2017). Gênese Instrumental do artefato simbólico função de uma variável real definida por várias sentenças matemáticas em um ambiente não digital. Revista Iberoamericana de Educación Matemática, (51), 107-125. Recuperado de http://www.fisem.org/ www/union/revistas/2017/51/05.pdf 